\title{
Foliations Associated to Regular Poisson Structures
}

\author{
Mélanie Bertelson *
}

October 24, 2018

\begin{abstract}
A regular Poisson manifold can be described as a foliated space carrying a tangentially symplectic form. Examples of foliations are produced here that are not induced by any Poisson structure although all the basic obstructions vanish.
\end{abstract}

\section{Introduction}

A Poisson manifold is a smooth manifold $M$ whose algebra of functions $C^{\infty}(M)$ carries a Lie algebra bracket $\{$,$\} for which each adjoint operator \{f, \cdot\}$ is a derivation of the pointwise multiplication. As revealed by an investigation of its local geometry, a Poisson manifold has a natural (possibly singular) foliation whose leaves are symplectic manifolds. Conversely, this "symplectic foliation" determines the Poisson structure. When the foliation is regular, the symplectic structures on the leaves assemble into a section of the second exterior power of the cotangent bundle to the foliation, called a leafwise symplectic structure.

This work addresses the problem of characterizing regular foliations that arise from Poisson structures in this fashion. One motivation for this topic is that it constitutes a first step towards classifying Poisson manifolds (cf. [9]). More heuristically, any problem in Poisson geometry potentially splits into two problems : one of them concerns foliations; the other one concerns leafwise symplectic structures. From this perspective, it is important to understand the relationship between the two. In particular, investigating the map \{Poisson structures $\} \stackrel{\varphi}{\rightarrow}$ \{foliations $\}$ is of special relevance.

The question of existence of symplectic structures on manifolds appears as the special case where the foliation has a single leaf. For closed manifolds, this problem is very difficult and mostly unsolved, although a lot of progress has recently been made. In particular, Taubes, using Seiberg-Witten theory, exhibits much subtler obstructions than the basic ones (a de Rham class whose

\footnotetext{
*Max-Planck-Institut für Mathematik, D-53111 Bonn, Germany (bertel@mpimbonn.mpg.de). This work has been supported by an Alfred P. Sloan Dissertation Fellowship.
} 
exterior powers do not vanish, and a compatible nondegenerate 2-form) [8]. On the other hand, for open manifolds, Gromov showed in 1969 that the parametric h-principle for open, invariant relations is valid [5, 6]. This result implies that on an open manifold, every nondegenerate 2 -form is homotopic to a symplectic form, and therefore reduces the problem to a question of obstruction theory.

Our purpose is to understand how far this result can be generalized to foliated manifolds. In particular, we would like to find a notion of "openness" for such spaces that is natural and broad enough, and for which the following statement holds : on an open foliated manifold every leafwise nondegenerate 2 -form is homotopic to a leafwise symplectic form. Henceforth, a foliated manifold with noncompact leaves for which this statement holds will be said to be "open" (provided the statement is nonempty, that is, leafwise nondegenerate 2 -forms exist). The requirement that the leaves be noncompact is not sufficient to ensure that the foliation is "open", but implies, together with the existence of a leafwise nondegenerate 2 -form, that each leaf admits its own symplectic structure. Locally trivial fibrations are "open" when their fibers are noncompact. This is implied by Gromov's h-principle mentioned above.

Examples of non- "open" foliated manifolds are produced here. Although the foliations exhibited carry leafwise nondegenerate 2 -forms, and in some cases display no identifiable closedness feature, they do not carry any leafwise symplectic form. An intriguing example consists of a foliation associated to a submersion whose fibers are all noncompact, connected, and diffeomorphic to one another. It is not a locally trivial fibration of course, but seems very close to being one. These examples show that one cannot be too optimistic about the size of the class of "open" foliated manifolds, and they illustrate different types of simple obstructions that one may encounter when trying to build leafwise symplectic structures.

In a subsequent paper [2] (see also [1]), the following theorem is proved: $O n$ a foliated manifold $(M, \mathcal{F})$ that admits a positive, proper function $f: M \rightarrow \mathbb{R}$, without leafwise local maxima, satisfying certain generic nondegeneracy conditions, the h-principle for open and foliated-invariant relations is valid. In particular, under the hypotheses of this theorem, every leafwise nondegenerate 2 -form is homotopic to a leafwise symplectic form. The condition of existence of such a function seems thus to be a good candidate for the definition of open foliated manifold, all the more, since it corresponds to the usual definition in the case of foliations with a single leaf. Besides, it is not difficult to verify directly that the foliated manifolds described here do not support proper functions without leafwise local maxima.

Acknowledgements. I wish to thank Alan Weinstein for suggesting this problem and for constant and helpful advice, as well as Yasha Eliashberg for many insightful conversations.

\section{Preliminaries}




\section{$1.1 \quad$ Regular Poisson manifolds}

The definition of regular Poisson manifolds, and the existence of the associated symplectic foliation are recalled in this section.

Definition 1.1 A Poisson manifold is a smooth manifold whose algebra of functions $C^{\infty}(M)$ is endowed with a Lie bracket $\{$,$\} acting on each argument as a$ derivation, that is, the following identity (called Leibniz identity) is satisfied for all $f, g$ and $h$ in $C^{\infty}(M)$ :

$$
\{f g, h\}=g\{f, h\}+f\{g, h\} .
$$

As a consequence of this definition, a vector field $X_{f}=\{f, \cdot\}$ is associated to each function $f \in C^{\infty}(M)$. Moreover, the map $C^{\infty}(M) \rightarrow \Gamma T M: f \mapsto X_{f}$ is a Lie algebra homomorphism.

Definition 1.2 When the rank of the distribution $\mathcal{D}=\left\{X_{f}(x) ; f \in C^{\infty}(M), x \in\right.$ $M\}$ is constant, the Poisson manifold is said to be regular.

A regular Poisson manifold supports a foliation whose leaves are symplectic manifolds. Indeed, the distribution $\mathcal{D}$ is involutive, thus integrable, and a leaf $F$ carries the symplectic structure $\omega_{F}$ defined by the following expression :

$$
\omega_{F}\left(\left.X_{f}\right|_{F},\left.X_{g}\right|_{F}\right)=\left.\{d f, d g\}\right|_{F} \quad f, g \in C^{\infty}(M) .
$$

The symplectic structures along the leaves assemble into a global section of the bundle $\Lambda^{2} T^{*} \mathcal{F}$. (Here $T \mathcal{F}$ denotes the tangent bundle to the foliation, and $T^{*} \mathcal{F}$ denotes its dual.) Conversely, the data of a foliation $\mathcal{F}$, endowed with a section $\omega$ of the bundle $\Lambda^{2} T^{*} \mathcal{F}$ whose restriction to each leaf is a symplectic form, determines a regular Poisson structure having $\mathcal{F}$ as associated foliation.

\subsection{Tangential de Rham cohomology}

This section introduces natural extensions to foliated spaces of the complex of differential forms and de Rham cohomology. This material can be found in [7] for example.

Let $\Omega^{k}(\mathcal{F})$ denote the space of sections of the bundle $\Lambda^{k} T^{*} \mathcal{F}$. The elements of $\Omega^{k}(\mathcal{F})$ are called tangential differential $k$-forms, and can be thought of as skewsymmetric $C^{\infty}(M)$-multilinear maps $\Gamma T \mathcal{F} \times \ldots \times \Gamma T \mathcal{F} \rightarrow C^{\infty}(M)$. As for ordinary differential forms, the expression

$$
\begin{aligned}
d_{\mathcal{F}} \alpha\left(X_{0}, \ldots, X_{k}\right)= & \sum_{i=0}^{k}(-1)^{i} X_{i}\left(\alpha\left(X_{0}, \ldots, \hat{X}_{i}, \ldots, X_{k}\right)\right)+ \\
& \sum_{i<j}(-1)^{i+j} \alpha\left(\left[X_{i}, X_{j}\right], X_{0}, \ldots, \hat{X}_{i}, \ldots, \hat{X}_{j}, \ldots, X_{k}\right),
\end{aligned}
$$


where $\alpha \in \Omega^{k}(\mathcal{F})$, where $X_{0}, \ldots, X_{k} \in \Gamma T \mathcal{F}$, and where a symbol covered by a hat is omitted, defines a degree one differential operator $d_{\mathcal{F}}$ that satisfies $d_{\mathcal{F}}{ }^{2}=0$. A tangential differential $k$-form $\alpha$ with $d_{\mathcal{F}} \alpha=0$ is said to be $d_{\mathcal{F}}$ closed, and if $\alpha=d_{\mathcal{F}} \beta$ for some tangential differential $(k-1)$-form $\beta$, then $\alpha$ is

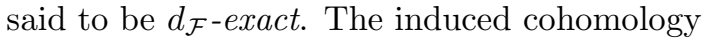

$$
H^{\star}(\mathcal{F})=\frac{\left\{d_{\mathcal{F}} \text {-closed } \star \text {-forms }\right\}}{\left\{d_{\mathcal{F}} \text {-exact } \star \text {-forms }\right\}}
$$

is called the tangential de Rham cohomology.

The natural inclusion $i: T \mathcal{F} \rightarrow T M$ induces surjective bundle maps $r_{k}$ : $\Lambda^{k} T^{*} M \rightarrow \Lambda^{k} T^{*} \mathcal{F}$. In particular, any tangential differential form $\alpha$ is the restriction to $\Gamma T \mathcal{F} \times \ldots \times \Gamma T \mathcal{F}$ of some ordinary differential form, called hereafter an extension of $\alpha$.

Remark 1.3 If the foliation has a single leaf, we recover the ordinary de Rham cohomology.

The following two properties (Proposition 1.4 and Proposition 1.5) will be useful in the next section, where calculation of the tangential de Rham cohomology for some specific foliations will be needed.

Proposition 1.4 ([3]) (Mayer-Vietoris sequence.) Let $(M, \mathcal{F})$ be a foliated manifold, and let $U$ and $V$ be open subsets of $M$. Then the following short sequence is exact :

$$
\begin{aligned}
& 0 \rightarrow \Omega^{k}\left(\left.\mathcal{F}\right|_{U \cup V}\right) \rightarrow \Omega^{k}\left(\left.\mathcal{F}\right|_{U}\right) \oplus \Omega^{k}\left(\left.\mathcal{F}\right|_{V}\right) \rightarrow \Omega^{k}\left(\left.F\right|_{U \cap V}\right) \rightarrow 0 \\
& \alpha \quad \mapsto \quad\left(\left.\alpha\right|_{U},\left.\alpha\right|_{V}\right) \\
& \left.(\beta, \gamma) \quad \mapsto \gamma\right|_{U \cap V}-\left.\beta\right|_{U \cap V} .
\end{aligned}
$$

It therefore induces a long exact sequence in cohomology :

$$
\ldots \rightarrow H^{k}\left(\left.\mathcal{F}\right|_{U \cup V}\right) \rightarrow H^{k}\left(\left.\mathcal{F}\right|_{U}\right) \oplus H^{k}\left(\left.\mathcal{F}\right|_{V}\right) \rightarrow H^{k}\left(\left.F\right|_{U \cap V}\right) \rightarrow H^{k+1}\left(\left.\mathcal{F}\right|_{U \cup V}\right) \rightarrow \ldots
$$

The proof is similar to that of exactness of the ordinary Mayer-Vietoris sequence in de Rham cohomology.

Let $\left(M_{1}, \mathcal{F}_{1}\right)$ and $\left(M_{2}, \mathcal{F}_{2}\right)$ be two foliated manifolds, and consider their Cartesian product $\left(M_{1} \times M_{2}, \mathcal{F}_{1} \times \mathcal{F}_{2}\right)$. For every pair $(i, j)$ of integers, there is a pairing

$$
\begin{aligned}
\times_{i j}: \Omega^{i}\left(\mathcal{F}_{1}\right) \otimes \Omega^{j}\left(\mathcal{F}_{2}\right) & \rightarrow \Omega^{i+j}\left(\mathcal{F}_{1} \times \mathcal{F}_{2}\right) \\
\left(\alpha_{1} \otimes \alpha_{2}\right) & \mapsto p_{1}^{*}\left(\alpha_{1}\right) \wedge p_{2}^{*}\left(\alpha_{2}\right)
\end{aligned}
$$

where $p_{\ell}$ denotes the natural projection of $M_{1} \times M_{2}$ onto its $\ell$-th factor. These pairings induce maps in cohomology :

$$
\times^{k}: \bigoplus_{i+j=k} H^{i}\left(\mathcal{F}_{1}\right) \otimes H^{j}\left(\mathcal{F}_{2}\right) \rightarrow H^{k}\left(\mathcal{F}_{1} \times \mathcal{F}_{2}\right) .
$$


When the map $x^{k}$ is an isomorphism, we say that the Künneth formula

$$
H^{\star}\left(\mathcal{F}_{1} \times \mathcal{F}_{2}\right) \simeq \bigoplus_{i+j=\star} H^{i}\left(\mathcal{F}_{1}\right) \otimes H^{j}\left(\mathcal{F}_{2}\right)
$$

is valid. The following result will be sufficient for our needs.

Proposition 1.5 (迎) Let $\left(M_{1}, \mathcal{F}_{1}\right)$ be any foliated manifold, and let $M_{2}$ be a manifold of finite type foliated by a single leaf. Then the Künneth formula associated to the product $\left(M_{1}, \mathcal{F}_{1}\right) \times M_{2}$ is valid, that is,

$$
H^{k}\left(\mathcal{F}_{1} \times M_{2}\right) \simeq \bigoplus_{i+j=k} H^{i}\left(\mathcal{F}_{1}\right) \otimes H^{j}\left(M_{2}\right) \quad \text { for all } k .
$$

The proof is again very similar to the proof of the corresponding result in the single-leaf case.

\subsection{Terminology and first examples}

Definitions 1.6 Let $(M, \mathcal{F})$ be a foliated manifold.

- A leafwise almost symplectic form is a tangential differential 2-form $\beta$ that is nondegenerate, that is, the bundle map $T \mathcal{F} \rightarrow T^{*} \mathcal{F}: X \mapsto \beta(X, \cdot)$ is an isomorphism.

- A leafwise symplectic form is a $d_{\mathcal{F}}$-closed, nondegenerate, tangential differential 2-form. Equivalently, it is a tangential differential 2-form whose restriction to each leaf is a symplectic form.

- A leafwise volume form is a nonvanishing tangential differential form whose degree coincides with the dimension of the foliation. The foliated manifold $(M, \mathcal{F})$ is said to be orientable if it admits a leafwise volume form.

With this terminology, the data of a regular Poisson structure on a manifold is equivalent to that of a foliation endowed with a leafwise symplectic form.

Examples 1.7 (Examples of regular Poisson structures.)

a) A symplectic manifold is a regular Poisson manifold. Any manifold endowed with the trivial Poisson bracket $P=0$ is Poisson manifold as well.

b) Let $M$ be a manifold endowed with a two-dimensional foliation $\mathcal{F}$, and suppose that $(M, \mathcal{F})$ is orientable. Then any leafwise volume form is a leafwise symplectic form (and vice versa). The set of leafwise symplectic structures is therefore parameterized by the set of nonvanishing smooth functions. 
c) Let $(M, P)$ be any Poisson manifold. The rank of $P$ is maximum on $O_{1}$, an open subset of $M$. Similarly, the rank of $\left.P\right|_{M-\bar{O}_{1}}$ is maximum on an open subset $\mathrm{O}_{2}$, and so forth. This shows that $M$ contains a dense open set $O=\cup_{i} O_{i}$ such that $\left(O,\left.P\right|_{O}\right)$ is a disjoint union of finitely many regular Poisson manifolds.

d) As explained in the next section, a locally trivial fibration with noncompact fibers that admits a leafwise almost symplectic form also admits a leafwise symplectic form.

e) More examples can be constructed by means of the criterion for existence of leafwise symplectic structure exhibited in [2.

Remark 1.8 As illustrated by the following example, a compact manifold may

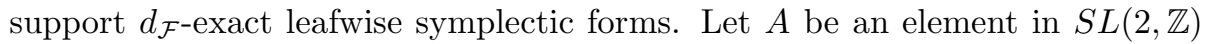
with $\operatorname{trace}(A)>2$. Consider the foliated manifold $(M, \mathcal{F})$ constructed by suspension of $A$, thought of as an isomorphism of the foliated manifold $\left(\mathbb{T}^{2}, \mathcal{F}_{X}\right)$, where $\mathcal{F}_{X}$ denotes the (irrational slope) linear foliation of $\mathbb{T}^{2}$ in the direction of an eigenvector $X$ of $A$. It is shown in [3] that the second tangential de Rham cohomology space of $\mathcal{F}_{X}$ vanishes; the leafwise symplectic structures on $(M, \mathcal{F})$ are therefore all exact.

\subsection{Description of the problem}

Given a manifold $M$, consider the map

$$
\begin{gathered}
\{\text { rank- } 2 k \text { regular Poisson structures on } M\} \\
\downarrow \varphi \\
\{2 k \text {-dimensional foliations on } M\} .
\end{gathered}
$$

As explained in the introduction, the problem approached in this work is that of describing the image of this map. More specifically, we aim at finding interesting necessary and sufficient conditions on a foliation $\mathcal{F}$ to be in the image of $\varphi$. Along those lines, the following observations are relevant.

\section{Observations 1.9}

(I) A foliation $\mathcal{F}$ supporting a leafwise symplectic form obviously satisfies the following conditions.

i) Each leaf of $\mathcal{F}$ supports some symplectic form.

ii) The foliated manifold $(M, \mathcal{F})$ supports some leafwise almost symplectic form (Definition 1.6). Equivalently, $T \mathcal{F}$ admits a structure of symplectic vector bundle. In particular, the foliated manifold $(M, \mathcal{F})$ is orientable.

(II) For foliations with a single leaf, the problem becomes that of characterizing manifolds supporting some symplectic form. There is a strong dichotomy between open and closed manifolds. For closed manifolds, the existence problem is hard and essentially unsolved. The basic obstructions are : 
a) Existence of a class $a$ in the second de Rham cohomology space of $M$ such that $a^{k} \neq 0$, where $k=\frac{1}{2} \operatorname{dim} M$.

b) Existence of an almost symplectic structure or equivalently of an almost complex structure inducing a volume compatible with $a^{k}$.

Results of Taubes involving Seiberg-Witten theory imply that fulfillment of these two conditions does not guarantee existence of a symplectic form. For instance, the connected sum of three copies of $\mathbb{C} P^{2}$ satisfies conditions a) and b), but does not carry any symplectic form $([8])$. For open manifolds, when one does not require the symplectic structure to have any particular behavior at infinity or at the boundary, the problem has been solved by Gromov in his thesis. He proved the following result.

Theorem 1.10 (河) On an open manifold $M$, any almost symplectic form is homotopic among almost symplectic forms to a symplectic form.

(III) The next simplest foliations are locally trivial fibrations. Theorem 1.10 (or rather a stronger version of this theorem) implies the following result.

Corollary 1.11 (泃) Let $\pi: M \rightarrow B$ be a locally trivial fibration whose fiber is an open manifold $F$. Then any leafwise almost symplectic form on $(M, \mathcal{F})$ is homotopic, among such forms, to a leafwise symplectic form.

(IV) In view of Theorem 1.10, if the foliation at hand has no compact leaf, then property ii) above implies property i).

Our approach has been to focus on foliations with noncompact leaves, and search for a generalization of Theorem 1.10 alongside guiding examples. It is important to keep in mind that one does not control the behavior at infinity of the symplectic structure constructed in the proof of Theorem 1.10. In contrast, a symplectic structure on a leaf of a foliation that extends to a leafwise symplectic structure on the entire foliated manifold is most likely very constrained at infinity, due partly to recurrence phenomena, partly to the influence of neighboring symplectic leaves. We have been trying to grasp the type of behavior at infinity that a foliation supporting a leafwise symplectic structure typically has. The examples in Section 2 provide some answers to natural questions directed along those lines. A generalization of Theorem 1.10 will appear in [2]. Its statement is as follows :

Theorem $1.12([2])$ Let $(M, \mathcal{F})$ be an open foliated manifold. Then any leafwise almost symplectic form is homotopic, among leafwise almost symplectic forms, to a leafwise symplectic form.

An open foliation manifold is defined to be foliated manifold that admits a positive, proper function, without leafwise local maxima, whose jet satisfies some transversality condition (see [2, 1] for a precise definition). 
Remark 1.13 Let $(M, \mathcal{F})$ be a foliated manifold that admits a proper function $f: M \rightarrow[0, \infty)$ without leafwise local maxima. Consider a partition $\mathcal{P}=$ $\left\{a_{0}=0<a_{1}<\ldots<a_{k}<\ldots\right\}$ of $[0, \infty)$ by noncritical values of $f$, and the associated exhaustion of $M$ by the slices $K_{i}=f^{-1}\left(\left[a_{i}, a_{i+1}\right]\right)$. One observes that the condition that $f$ has no leafwise local maxima implies that for any leaf $F$ of the foliation $\mathcal{F}$, and for any index $i$, the set $F-\operatorname{int}\left(K_{i}\right)$ has no compact connected component $\left(\left.f\right|_{F}\right.$ would necessarily achieve a local maximum on such a component).

Using this criterion, it is easy to verify directly (that is, independently of Theorem 1.12) that the foliated manifolds constructed in the next section are not open.

\section{Examples}

This section could be entitled variations on the theme:

a compact manifold with vanishing second de Rham cohomology is never symplectic.

It presents examples of foliated manifolds with noncompact leaves that do not carry any leafwise symplectic structure, although leafwise almost symplectic structures exist. For all of them, the second tangential de Rham cohomology space vanishes, so that a leafwise symplectic structure would necessarily be exact, and the argument consists in proving that the foliated manifold at hand may not carry any exact leafwise symplectic structure. In each case, the leaves being noncompact, the argument is not as simple as that of the theme, but presents nevertheless a strong analogy with it. Therefore, it seems tempting, in view of Theorem 1.10, to attribute nonexistence of exact leafwise symplectic structures to some kind of compactness, or non-openness, in disguise. We have followed this line of ideas through the exposition of the examples, trying to provide some interpretation for each.

The exposition is organized as follows. The examples are presented along with arguments for nonexistence of exact leafwise symplectic structures. There is not a one to one correspondence between the two. The arguments are independent, but the examples are not really so. They evolve from seemingly quite closed to seemingly more and more open, so that, as already mentioned earlier, the last one is the most seemingly open one and therefore the most interesting one too. Nevertheless, we felt it worthwhile to include the previous examples as well.

\subsection{Leafwise almost symplectic structures}

We begin with showing a simple conditions ensuring existence of a leafwise almost symplectic structure (Definition 1.6). 
Lemma 2.1 A foliated manifold of the type $(N, \mathcal{G}) \times P$, where $\mathcal{G}$ is the foliation of $N$ by the orbits of a nonvanishing vector field $X$, and where $P$ is an odddimensional manifold that admits a contact form, always supports some leafwise almost symplectic structure.

Recall that a contact form is a 1 -form $\alpha$ such that $\alpha \wedge(d \alpha)^{k}$ does not vanish for $k=\frac{1}{2}(\operatorname{dim} P-1)$.

Proof. Let $\beta$ be a 1 -form on $N$ such that $\beta(X)=1$. Then $\beta \wedge \alpha+d \alpha$ extends a leafwise almost symplectic form.

More generally, define a leafwise almost contact structure on a manifold endowed with a $(2 k+1)$-dimensional foliation to be a pair $(\alpha, \beta)$, consisting of a tangential differential 1-form $\alpha$ and a tangential differential 2 -form $\beta$, such that $\alpha \wedge(\beta)^{k}$ is leafwise volume form. If $\left(M_{1}, \mathcal{F}_{1}\right)$ and $\left(M_{2}, \mathcal{F}_{2}\right)$ admit leafwise almost contact structures $\left(\alpha_{1}, \beta_{1}\right)$ and $\left(\alpha_{2}, \beta_{2}\right)$ respectively, then $\beta_{1}+\alpha_{1} \wedge \alpha_{2}+\beta_{2}$ is a leafwise almost symplectic structure on $\left(M_{1} \times M_{2}, \mathcal{F}_{1} \times \mathcal{F}_{2}\right)$.

\subsection{First variation}

Let $a$ be an irrational number, and consider the foliated manifold $\left(\mathbb{T}^{2}, \mathcal{F}_{a}\right) \times S^{3}$, where $\mathcal{F}_{a}$ denotes the linear foliation of $\mathbb{T}^{2}$ of slope $a$. It admits a leafwise almost symplectic structure (Lemma 2.1), but not any leafwise symplectic structure. The latter statement is, as will be seen below, a consequence of the following proposition.

Proposition 2.2 A foliated manifold $(M, \mathcal{F})$ satisfying the following two conditions does not support any exact leafwise symplectic structure.

- $M$ is compact,

- $(M, \mathcal{F})$ admits a transverse volume form $\mu$.

Let us recall that a transverse volume form on a manifold $M$, endowed with a foliation $\mathcal{F}$ of codimension $q$, is a nonvanishing closed $q$-form $\mu$ such that $i(X) \mu=0$ for any vector field $X$ tangent to $\mathcal{F}$. It is easily seen that the foliated manifold $\left(\mathbb{T}^{2}, \mathcal{F}_{a}\right) \times S^{3}$ admits such a form. (If $\theta$ and $\varphi$ are coordinates on $\mathbb{T}^{2}$ for which the vector field $\frac{\partial}{\partial \theta}+a \frac{\partial}{\partial \varphi}$ is tangent to the foliation $\mathcal{F}_{a}$, the form

$$
\mu=p_{1}^{*}(a d \theta-d \varphi),
$$

where $p_{1}$ is the natural projection of $\mathbb{T}^{2} \times S^{3}$ onto its first factor, is a transverse volume form.) Observe that, as implied by Proposition 1.5, $H^{2}\left(\mathcal{F}_{a} \times S^{3}\right)=0$. (Notice that the manifold $S^{3}$ could be replaced by any homology three-sphere, or any odd-dimensional manifold $P$ endowed with an almost contact structure and satisfying $H^{1}(P)=0=H^{2}(P)$ ). 
Proof of Proposition 2.9. Suppose on the contrary that $(M, \mathcal{F})$ admits an exact leafwise symplectic structure $\omega=d_{\mathcal{F}} \alpha$. Let $\tilde{\omega} \in \Omega^{2}(M)$ be an extension of $\omega$. Then the form $(\tilde{\omega})^{k} \wedge \mu$ is a volume form on $M$. We claim that $(\tilde{\omega})^{k} \wedge \mu$ is exact. Indeed, $(\tilde{\omega})^{k} \wedge \mu=d\left(\tilde{\alpha} \wedge(\tilde{\omega})^{k-1} \wedge \mu\right)$, where $\tilde{\alpha}$ is an extension of $\alpha$.

\subsection{Second variation}

In the previous example, closedness of the ambient manifold plays, it seems, a crucial role. Besides, it is to be expected that a foliation on a closed manifold presents some features of a compact nature, and that a definition of openness for foliations would require the ambient manifold to be nonclosed. Nevertheless, compactness of the ambient manifold is not, as will be seen, the only missing obstruction to existence of a leafwise symplectic structure.

Starting from the preceding example $\left(\mathbb{T}^{2}, \mathcal{F}_{a}\right) \times S^{3}$ and removing a point $p$ from $\mathbb{T}^{2}$ provides a noncompact foliated manifold $(M, \mathcal{F})=\left(\mathbb{T}^{2}-\{p\},\left.\mathcal{F}_{a}\right|_{\mathbb{T}^{2}-\{p\}}\right)$ $\times S^{3}$ with open leaves (Figure 1) that admits a leafwise almost symplectic structure (since $\left(\mathbb{T}^{2}, \mathcal{F}_{a}\right) \times S^{3}$ already did). Nevertheless, the foliated manifold $(M, \mathcal{F})$ does not support any leafwise symplectic structure. This is a consequence of the following proposition, combined with the fact that $H^{2}(\mathcal{F})$ vanishes (as implied by Proposition 1.5).

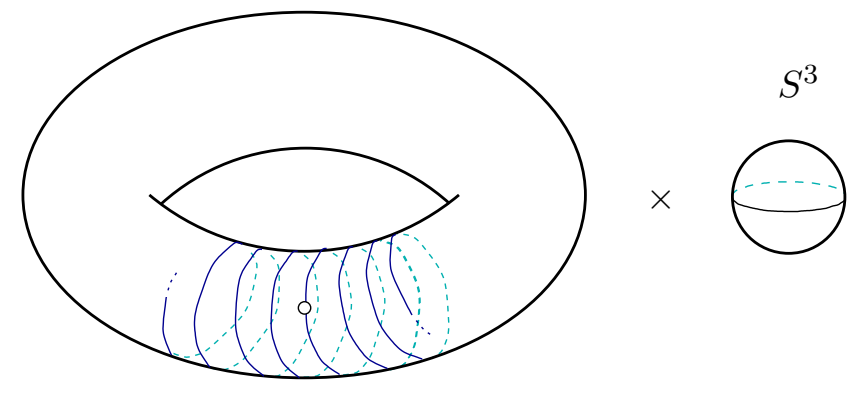

Figure 1: The foliated manifold $\left(\mathbb{T}^{2}-\{p\},\left.\mathcal{F}_{a}\right|_{\mathbb{T}^{2}-\{p\}}\right) \times S^{3}$

Proposition 2.3 A foliated manifold $(M, \mathcal{F})$ of the type $(N, \mathcal{G}) \times P$, where $P$ is any closed odd-dimensional manifold, and where $\mathcal{G}$ is the foliation of $N$ by the orbits of a nonvanishing vector field $X$ admitting some nonclosed orbit, may not support any exact leafwise symplectic structure.

Proof. Suppose that $\alpha$ is a tangential differential 1-form on $(M, \mathcal{F})$ for which $d_{\mathcal{F}} \alpha=\omega$ is a leafwise symplectic structure. Let $2 k=\operatorname{dim} \mathcal{F}$. If $i: S \rightarrow M$ is an immersed oriented submanifold of dimension $2 k-1$ contained in a leaf $F$, define

$$
\nu(S)=\int_{S} i^{*}\left(\alpha \wedge \omega^{k-1}\right) .
$$


Observe that if $S$ bounds a compact domain $D$ in $F$, then

$$
\nu(S)= \pm \int_{D} \omega^{k} .
$$

We show that the symplectic volume of a nonclosed leaf $F$ of $\mathcal{F}$ is necessarily infinite. Let $a$ be an accumulation point of the leaf $F$ that does not lie in $F$, and let $(U, \varphi)$ be a chart adapted to $\mathcal{F}$ and centered at $a$. For $x$ in $U$, denote by $P_{x}$ the plaque of $\left.\mathcal{F}\right|_{U}$ containing $x$. Consider a sequence $\left(a_{i}\right)$ contained in $U \cap F$ that converges to $a$. Let $\delta$ be the symplectic volume of the plaque $P_{a}$. Then, for $i$ sufficiently large, the symplectic volume of the plaque $P_{a_{i}}$ is at least $\frac{\delta}{2}$. We have thus infinitely many disjoint subsets of $F$, each which contributes at least $\frac{\delta}{2}$ to the volume of $F$. The latter must therefore be infinite. Notice that this argument shows that the volume of the end $\mathcal{E}$ of $F$ containing the sequence $\left(a_{i}\right)$ is infinite as well.

On the other hand, the volume of $\mathcal{E}$, or rather of a set $U \simeq[0, \infty) \times P$ representing $\mathcal{E}$, can be written as follows :

$$
\begin{aligned}
\lim _{n \rightarrow \infty} \int_{[0, n] \times P} \frac{\omega^{k}}{k !} & =\lim _{n \rightarrow \infty} \int_{\partial[0, n] \times P} \frac{1}{k !} \alpha \wedge \omega^{k-1} \\
& =\lim _{n \rightarrow \infty} \frac{1}{k !}(\nu(\{n\} \times P)-\nu(\{0\} \times P)),
\end{aligned}
$$

where $\{t\} \times P$ is oriented as the boundary of $[0, t] \times P$, the latter being oriented by the leafwise symplectic form $\omega$. Because the volume of $\mathcal{E}$ is infinite, $\lim _{n \rightarrow \infty} \nu(\{n\} \times P)=\infty$. But the map

$$
N \rightarrow \mathbb{R}: q \mapsto \nu(\{q\} \times P)
$$

is continuous (even smooth). Therefore, if $q_{a}$ denotes the first coordinate of $a$ with respect to the splitting $M=N \times P$, the quantity $\nu\left(\left\{q_{a}\right\} \times P\right)$ has to be infinite, and we reach a contradiction.

Remark 2.4 Observe that if some orbit $\varphi^{\mathbb{R}}(x)$ of the vector field $X$ is contained in its positive (or negative) $\operatorname{limit}_{\text {set }} \lim _{t \rightarrow \infty} \varphi^{t}(x)$ (as is the case for a vector field tangent to the foliation $\left(\mathbb{T}^{2}-\{p\},\left.\mathcal{F}_{a}\right|_{\mathbb{T}^{2}-\{p\}}\right)$ ), the conclusion of Proposition 2.3 can be strengthened as follows : the foliation $(M, \mathcal{F})$ may not support any leafwise symplectic structure whose restriction to the leaf $F=\varphi^{\mathbb{R}}(x) \times P$ is an exact form. Therefore, to obtain nonexistence of leafwise symplectic structures on such a foliation, it is sufficient that the second de Rham cohomology of the leaf $F$ vanishes. In particular, the foliated manifold $\left(\mathbb{T}^{2}-\{p\},\left.\mathcal{F}_{a}\right|_{\mathbb{T}^{2}-\{p\}}\right) \times P$, where $P$ is a manifold with $H^{2}(P)=0$ does not carry any leafwise symplectic structure.

Remark 2.5 The above lemma also proves that the first example $\left(\mathbb{T}^{2}, \mathcal{F}_{a}\right) \times S^{3}$ does not carry any leafwise symplectic structure. More generally, it shows that 
a foliated manifold of the type considered in the previous proposition may not support any leafwise symplectic structure whose top exterior power is exact (simply replace $\alpha \wedge \omega^{k-1}$ in the proof of Proposition 2.3 by a primitive of $\wedge^{k} \omega$ ). This argument may also be generalized in another direction, namely to foliated manifolds having a leaf with a periodic end, that is, an end diffeomorphic to an infinite connected sum $P \# P \# \ldots$, where $P$, the period, is a manifold whose boundary splits into two diffeomorphic pieces. One has to pay attention, though, to the fact that the hypersurfaces along which the connected sum is performed (the $\{n\} \times P$ 's in the previous proof) might not "converge" in $M$ to a hypersurface in a leaf. Indeed, looking back at the Anosov foliation, described in Remark 1.8, one notices that its leaves are either planes or cylinders. Thus they have nonclosed periodic ends. Nevertheless, this foliation admits leafwise symplectic structures although its second tangential de Rham cohomology space vanishes. The argument used in the proof of Proposition 2.3 must therefore fail.

In the previous example, the leaves were not embedded, and it is understandable that this might create obstructions to existence of leafwise symplectic structures. Indeed, a nonembedded leaf carries an additional topology $\mathcal{T}$, that is coarser than the topology associated to its manifold structure. A symplectic form on such a leaf that is the restriction of a leafwise symplectic form would necessarily be in some sense continuous with respect to $\mathcal{T}$. It could just happen that some of the nonembedded leaves do not admit any symplectic structure possessing this additional property (this is the case for a leaf of the foliation $\left.\left(\mathbb{T}^{2}-\{p\},\left.\mathcal{F}_{a}\right|_{\mathbb{T}^{2}-\{p\}}\right) \times S^{3}\right)$. Nevertheless, Proposition 2.3 does not rely on the leaves being nonembedded, it also applies to some foliations with nonclosed embedded leaves, as illustrated by the following example. Thus, the presence of an additional topology is not the only problem either.

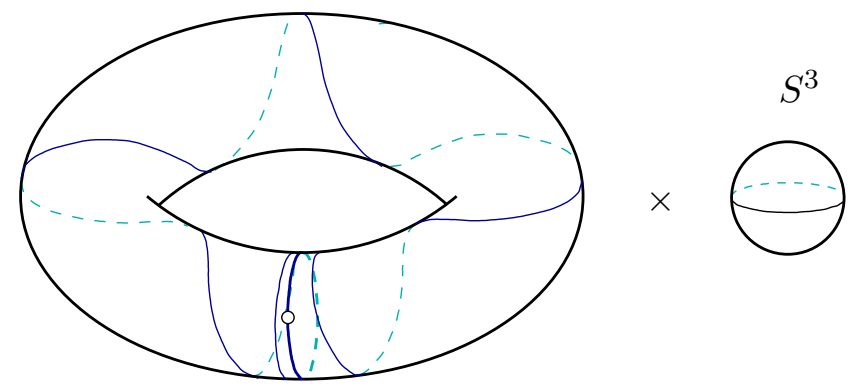

Figure 2: The foliated manifold $\left(\mathbb{T}^{2}-\{p\}, \mathcal{F}\right) \times S^{3}$

Consider the foliated manifold $(M, \mathcal{F})=\left(\mathbb{T}^{2}-\{p\}, \mathcal{F}\right) \times S^{3}$, where the foliation $\mathcal{F}$ is described as follows. Let $\theta$ and $\varphi$ be standard coordinates on $\mathbb{T}^{2}$ vanishing at $p$. Consider the vector field $X=\frac{\partial}{\partial \varphi}+f \frac{\partial}{\partial \theta}$ where $f$ is a smooth function on $\mathbb{T}^{2}$ such that 
- $f$ depends on $\theta$ only,

- $f(0)=0$,

- $f(\theta)>0$ for $\theta \neq 0$.

Let $\mathcal{G}$ denote the foliation of $\mathbb{T}^{2}$ by the orbits of $X$. It has one closed leaf $(\theta=0)$. The nonclosed leaves are embedded and accumulate on that closed leaf. The foliation we are interested in is $\mathcal{F}=p_{1}^{*}\left(\left.\mathcal{G}\right|_{\mathbb{T}^{2}-\{p\}}\right.$ ) (see Figure 2), where $p_{1}$ denotes the projection $\left(\mathbb{T}^{2}-\{p\}\right) \times S^{3} \rightarrow \mathbb{T}^{2}-\{p\}$. Because the point $p$ has been removed, the leaves of the foliation $\mathcal{F}$ are all noncompact. Here again, $H^{2}(\mathcal{F})$ vanishes (Proposition 1.5), and $(M, \mathcal{F})$ supports some leafwise almost symplectic structure (Lemma 2.1). However, Proposition 2.3 implies that $(M, \mathcal{F})$ does not support any leafwise symplectic structure.

\subsection{Third variation and main example}

We will present here an example of a foliation given by a submersion whose fibers are open, connected, and diffeomorphic to one another, that admits leafwise almost symplectic structures but not any leafwise symplectic structure. This foliation is not, of course, a locally trivial fibration (cf. Corollary 1.11), but seems nevertheless very close to being one.

Let $F$ be any closed manifold of dimension at least four with $H^{2}(F)=0$ that supports an almost symplectic structure (take $F=S^{1} \times S^{3}$ for instance). Consider the manifold $M^{\prime}=F \times(-1,1)$, the projection $p^{\prime}: M^{\prime} \rightarrow(-1,1)$, and the foliation $\mathcal{F}^{\prime}$ by the fibers of $p^{\prime}$. We will remove from $M^{\prime}$ a closed set $C$, consisting of two disjoint embedded lines, that intersects each leaf of $\mathcal{F}^{\prime}$ along exactly two points. Explicitly, let $p_{1}, q_{1}, p_{2}, q_{2}$ be four distinct points in $F$. Suppose that $p_{1}$ and $q_{1}$ (respectively $p_{2}$ and $q_{2}$ ) are contained in the interior of an embedded closed ball $U_{1}$ (respectively $U_{2}$ ), and that $U_{1} \cap U_{2}=\emptyset$. For $i=1,2$, let $\gamma_{i}:(-1,1) \rightarrow U_{i}$ be a path joining $p_{i}$ to $q_{i}$. Define $C$ as follows :

$$
C=\left(\bigcup_{t \in(-1,0]}\left\{\gamma_{1}(t), \gamma_{1}(-t)\right\} \times\{t\}\right) \cup\left(\bigcup_{t \in[0,1)}\left\{\gamma_{2}(t), \gamma_{2}(-t)\right\} \times\{t\}\right) .
$$

The foliated manifold $\left(M=M^{\prime}-C, \mathcal{F}=\left.\mathcal{F}^{\prime}\right|_{M^{\prime}-C}\right)$ (see Figure 3) admits a foliated almost symplectic structure. Its leaves are open, hence symplectic. But it does not support any leafwise symplectic structure. The proof below of the last assertion relies mostly on the vanishing of the second tangential de Rham cohomology space of $\mathcal{F}$.

\section{Lemma 2.6}

$$
H^{2}(\mathcal{F})=0
$$

Proof. For $i=1,2$, let $\tau_{i}$ be an open tubular neighborhood of $\partial U_{i}$ in $F$ such that if $U_{i}^{\prime}=U_{i} \cup \tau_{i}$ then $U_{1}^{\prime} \cap U_{2}^{\prime}=\emptyset$. Consider $W=M \cap\left(\left(U_{1}^{\prime} \cup U_{2}^{\prime}\right) \times(-1,1)\right)$ 


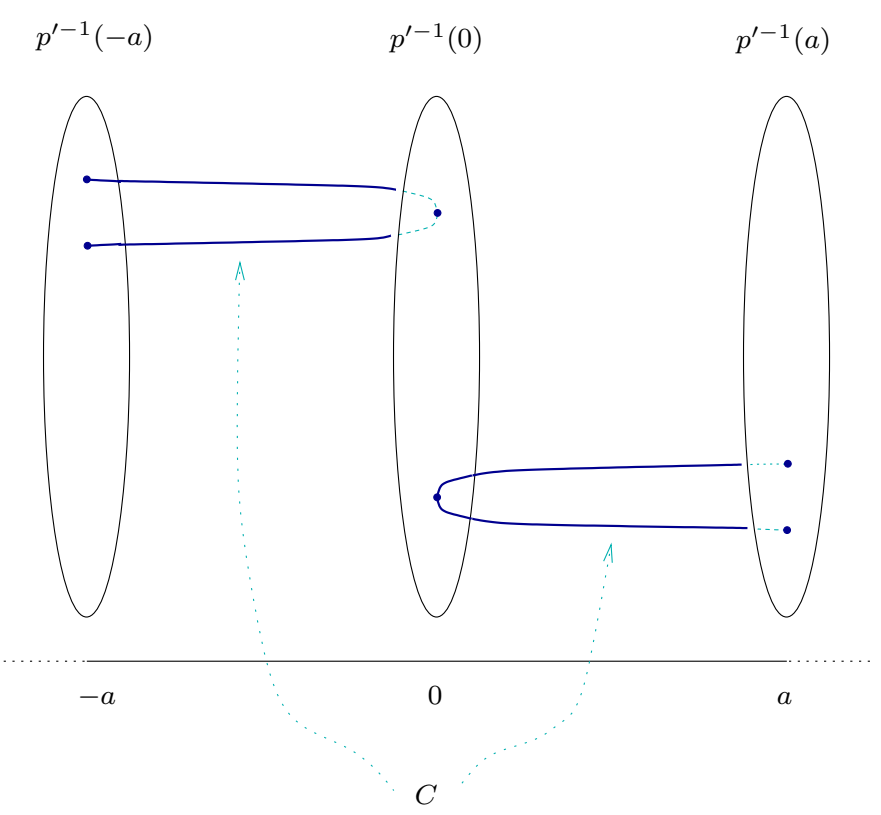

Figure 3: The curve $C$ sitting in $M^{\prime}$

and $W^{\prime}=M-\left(\left(U_{1} \cup U_{2}\right) \times(-1,1)\right)$. The Mayer-Vietoris sequence for the open subsets $W$ and $W^{\prime}$ (Proposition 1.4) contains this part

$\ldots \rightarrow H^{1}\left(\left.\mathcal{F}\right|_{W \cap W^{\prime}}\right) \rightarrow H^{2}(\mathcal{F}) \rightarrow H^{2}\left(\left.\mathcal{F}\right|_{W}\right) \oplus H^{2}\left(\left.\mathcal{F}\right|_{W^{\prime}}\right) \rightarrow H^{2}\left(\left.\mathcal{F}\right|_{W \cap W^{\prime}}\right) \rightarrow \ldots$

Since $\left.\mathcal{F}\right|_{W \cap W^{\prime}}$ and $\left.\mathcal{F}\right|_{W^{\prime}}$ are trivial foliations with leaves diffeomorphic to $\left(S^{3} \amalg S^{3}\right) \times \mathbb{R}$ and $F-\left(U_{1} \cup U_{2}\right)$ respectively, $H^{i}\left(\left.\mathcal{F}\right|_{W \cap W^{\prime}}\right)=0$ for $i=1,2$, and $H^{2}\left(\left.\mathcal{F}\right|_{W^{\prime}}\right)=0$ (cf. Lemma in [4] $)$. Hence $H^{2}(\mathcal{F})$ is isomorphic to $H^{2}\left(\left.\mathcal{F}\right|_{W}\right)$. On the other hand, the foliated manifold $\left(W,\left.\mathcal{F}\right|_{W}\right)$ is made of two disjoint pieces isomorphic to the following model.

Let $B^{n}$ (respectively $B_{r}^{n}$ ) denote the open ball in $\mathbb{R}^{n}$ of radius 1 (respectively $r)$. For $n \geq 4$, consider $N=B^{n} \times(-1,1)-C$, where $C=\cup_{t \in[0,1)}\{\gamma(t), \gamma(-t)\} \times$ $\{t\}$, and where $\gamma:(-1,1) \rightarrow B^{n}$ is a smooth path joining two distinct points in $B^{n}$. Suppose for convenience that $\gamma(0)=0$. Endow $N$ with the foliation $\mathcal{G}$ by the fibers of the natural projection $\pi: N \rightarrow(-1,1)$.

\section{Lemma 2.7}

$$
H^{2}(\mathcal{G})=0
$$

Proof. Let $\beta$ be a $d_{\mathcal{G}}$-closed tangential differential 2-form on $(N, \mathcal{G})$. Since $\mathcal{G}$ is trivial on $U^{-}=\pi^{-1}((-1,0))$ and on $U^{+}=\pi^{-1}((0,1))$, the Lemma in $\llbracket$ 
implies that there exists tangential differential 1-forms $\alpha^{-}$and $\alpha^{+}$, defined on $U^{-}$and $U^{+}$respectively, such that

$$
d_{\mathcal{G}} \alpha^{ \pm}=\left.\beta\right|_{U^{ \pm}} .
$$

Let $\phi_{1}$ and $\phi_{2}$ be smooth functions on $N$ such that

$$
\phi_{i}=\left\{\begin{array}{lll}
1 & \text { near } & \overline{O_{2 i}} \\
0 & \text { near } & N-O_{2 i-1}
\end{array}\right.
$$

where $O_{1}, O_{2}, O_{3}, O_{4}$ are open subsets of $N$ defined as follows :

$$
O_{j}=N \cap\left(\bigcup_{t \in(-1,1)} B_{\frac{|t|}{j}}^{n}(0) \times\{t\}\right) .
$$

We suppose that

$$
\{\gamma(t), \gamma(-t)\} \subset B_{\frac{|t|}{4}}^{n}(0) \text { for all } t \in(-1,1) .
$$

(If this is not true, replace in the definition of the $O_{j}$ 's the function $t \mapsto|t|$ by an appropriate continuous function vanishing at 0 ). Define

$$
\alpha=\left\{\begin{array}{ccl}
\phi_{1} \alpha^{-} & \text {on } & U^{-} \\
\phi_{1} \alpha^{+} & \text {on } & U^{+} \\
0 & \text { on } & \pi^{-1}(0) .
\end{array}\right.
$$

By construction, $\alpha$ is a smooth section of $T^{*} \mathcal{G}$, and the form $\beta^{\prime}=\beta-d_{\mathcal{G}} \alpha$ vanishes near $\overline{O_{2}}$. Let $\alpha^{\prime}$ be a tangential differential 1-form on $U=N-\overline{O_{4}}$ such that $d_{\mathcal{G}} \alpha^{\prime}=\left.\beta^{\prime}\right|_{U}$. The form $\alpha^{\prime}$ exists because the foliation $\left.\mathcal{G}\right|_{U}$ is isomorphic to a product foliation whose leaves are diffeomorphic to $B^{n}-\{0\}$. Since $\alpha^{\prime}$ is $d_{\mathcal{G}}$-closed on the open set $\tilde{U}=\left(O_{2}-\overline{O_{4}}\right)$, we have $\left.\alpha^{\prime}\right|_{\tilde{U}}=d_{\mathcal{G}} f$ for some smooth function $f$ defined on $\tilde{U}$. Let

$$
\alpha^{\prime \prime}=\left\{\begin{array}{cl}
\alpha^{\prime}-d_{\mathcal{G}}\left(\phi_{2} f\right) & \text { on } \tilde{U} \\
0 & \text { on } \overline{O_{4}} \\
\alpha^{\prime} & \text { elsewhere } .
\end{array}\right.
$$

The form $\alpha^{\prime \prime}$ is smooth and $d_{\mathcal{G}} \alpha^{\prime \prime}=\beta^{\prime}$. Thus $\beta=d_{\mathcal{G}}\left(\alpha^{\prime \prime}+\alpha\right)$.

End of the proof of Lemma 2.X.

This implies that $H^{2}\left(\left.\mathcal{F}\right|_{W}\right)=0$, hence that $H^{2}(\mathcal{F})=0$.

Proposition 2.8 The foliated manifold $(M, \mathcal{F})$ does not admit any leafwise symplectic structure. 
Proof. Suppose on the contrary that $\omega$ is a leafwise symplectic structure on $(M, \mathcal{F})$. As a consequence of Lemma 2.6, $\omega=d_{\mathcal{F}} \alpha$ for some tangential differential 1-form $\alpha$. Let $T$ denote the hypersurface $\partial U_{1} \times(-1,1)$. It separates $M$ into two domains $D^{1}$ and $D^{2}$, with $D^{1}$ contained in $U_{1} \times(-1,1)$. Let $p: M \rightarrow(-1,1)$ be the restriction of the map $p^{\prime}$ to $M$. For $i=1,2$ and for $t$ in $(-1,1)$, let $D_{t}^{i}=D^{i} \cap p^{-1}(t)$, let $T_{t}=T \cap p^{-1}(t)$, let $\omega_{t}=\left.\omega\right|_{p^{-1}(t)}$ and let $\alpha_{t}=\left.\alpha\right|_{p^{-1}(t)}$. For every negative $t, D_{t}^{2}$ is relatively compact and

$$
0<\int_{D_{t}^{2}} \omega_{t} \wedge \omega_{t}=\int_{\left(T_{t}, \circlearrowright\right)} \alpha_{t} \wedge \omega_{t} .
$$

The orientation implicitly assigned to $T_{t}$ is that induced from the volume form $\omega_{t} \wedge \omega_{t}$ when $T_{t}$ is thought of as the boundary of $D_{t}^{2}$. We denote this orientation by $\circlearrowright$. Similarly, for every positive $t$ we have

$$
0<\int_{D_{t}^{1}} \omega_{t} \wedge \omega_{t}=\int_{\left(T_{t}, \circlearrowleft\right)} \alpha_{t} \wedge \omega_{t}=-\int_{\left(T_{t}, \circlearrowright\right)} \alpha_{t} \wedge \omega_{t},
$$

where $\circlearrowleft$ denotes the opposite orientation on $T_{t}$. Besides, the map

$$
t \mapsto \int_{\left(T_{t}, \circlearrowright\right)} \alpha_{t} \wedge \omega_{t}
$$

is continuous. (Notice that until now we have only used the fact that each leaf of $\mathcal{F}$ had a trivial second de Rham cohomology. To be able to say that the function (3) is continuous, we need the stronger fact that $H^{2}(\mathcal{F})=0$.) It follows thus from (1) and (2) that

$$
\int_{T_{0}} \alpha_{0} \wedge \omega_{0}=0
$$

Now choose a closed ball $U_{1}^{\prime}$ embedded in $F$, containing $p_{1}$ and $q_{1}$, and such that $U_{1}^{\prime} \subset \operatorname{int}\left(U_{1}\right)$. The same argument applies to $U_{1}^{\prime}$ and shows that

$$
\int_{T_{0}^{\prime}} \alpha_{0} \wedge \omega_{0}=0
$$

for $T_{0}^{\prime}=\partial U_{1}^{\prime} \times\{0\}$. Observe that the hypersurface $T_{0} \cup T_{0}^{\prime}$ bounds in $F \times\{0\}$ a relatively compact domain $D_{0}=\left(\operatorname{int}\left(U_{1}\right)-U_{1}^{\prime}\right) \times\{0\}$. Moreover, we have

$$
0<\int_{D_{0}} \omega_{0} \wedge \omega_{0}=\int_{T_{0} \cup T_{0}^{\prime}} \alpha_{0} \wedge \omega_{0} .
$$

But (4) and (5) imply that the last expression vanishes, a contradiction.

\subsection{Fourth variation}

Let $M$ be an $(n+1)$-dimensional manifold endowed with a codimension one foliation $\mathcal{F}$ and a Riemannian metric $g$. 
Proposition 2.9 Suppose that the foliated manifold $(M, \mathcal{F})$ satisfies the following condition. For every positive function $\varepsilon: M \rightarrow(0, \infty)$, there exists a nonsymplectic $n$-dimensional submanifold $S$ of $M$ whose tangent space $T S$ is $\varepsilon$ close to $T \mathcal{F}$ (by which term is meant that $\|X-Y\|<\varepsilon(s)$ for every unit vectors $X$ and $Y$ in $T_{s} S$ and $T_{s} \mathcal{F}$ respectively and for every $s$ in $\left.S\right)$. Then $(M, \mathcal{F})$ does not support any leafwise symplectic structure that admits a closed extension.

Proof. Suppose, on the contrary, that $\Omega$ is a closed 2 -form on $M$ whose restriction to $T \mathcal{F} \times T \mathcal{F}$ is a leafwise symplectic structure. Consider the 1dimensional distribution $N_{\Omega} \mathcal{F}=\{X \in T M ; \Omega(X, \cdot)=0\}$. The closed form $\Omega$ restricts to a nondegenerate 2 -form, hence a symplectic form, on any hypersurface $S$ transverse to $N_{\Omega} \mathcal{F}$. Now let

$$
\varepsilon: M \rightarrow(0, \infty): x \mapsto d\left(T_{x} \mathcal{F},\left(N_{\Omega} \mathcal{F}\right)_{x}\right),
$$

where $d\left(T_{x} \mathcal{F},\left(N_{\Omega} \mathcal{F}\right)_{x}\right)=\inf \left\{\|X-Y\| ; X \in T_{x} \mathcal{F}, Y \in\left(N_{\Omega} \mathcal{F}\right)_{x}\right.$, and $\|X\|=$ $\|Y\|=1\}$. Any submanifold $S$ whose tangent space is $\varepsilon$-close to $T \mathcal{F}$ is necessarily transverse to $N_{\Omega} \mathcal{F}$, and therefore inherits a symplectic structure, contradicting the hypothesis.

Remark 2.10 Although the four different arguments for nonexistence of leafwise symplectic structures that have been presented here seem independent, it can be verified that the last one applies to all our examples. This does not remain true if we modify the examples so as to raise their codimension, which can be done without affecting existence of leafwise almost symplectic structures nor nonexistence of leafwise symplectic structures.

\section{References}

[1] M. Bertelson, Foliations associated to regular Poisson structures, Ph.D. Thesis, Stanford University, June 2000.

[2] M. Bertelson, An h-principle for certain open relations on foliated spaces. In preparation.

[3] A. El Kacimi-Alaoui, Sur la cohomologie feuilletée. (French) [On foliated cohomology] Compositio Math. 49 (1983), no. 2, 195-215.

[4] M.J. Gotay, R. Lashof, J. Śniatycki and A. Weinstein, Closed forms on symplectic fiber bundles. Comment. Math. Helv. 58 (1983), no. 4, 617621.

[5] M. Gromov, Stable mappings of foliations into manifolds. Izv. Akad. Nauk SSSR Ser. Mat. 33 (1969), 707-734.

[6] M. Gromov, Partial differential relations. Ergebnisse der Mathematik und ihrer Grenzgebiete (3) [Results in Mathematics and Related Areas (3)], 9. Springer-Verlag, Berlin-New York, 1986. 
[7] G. Hector, E. Macías, M. Saralegi, Lemme de Moser feuilleté et classification des variétés de Poisson régulières. (French) [A foliated version of Moser's lemma and classification of regular Poisson manifolds] Publ. Mat. 33 (1989), no. 3, 423-430.

[8] C.H. Taubes, The Seiberg-Witten invariants and symplectic forms. Math. Res. Lett. 1 (1994), no. 6, 809-822.

[9] A. Weinstein, Poisson geometry. Symplectic geometry, Differential Geom. Appl. 9 (1998), no. 1-2, 213-238. 